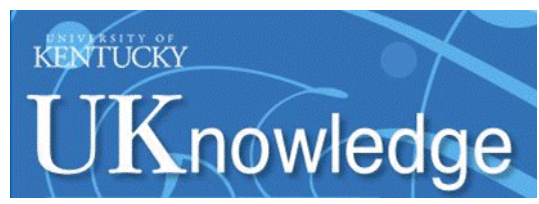

University of Kentucky

UKnowledge

6-1-1986

\title{
Radiation Pressure and Emission Clouds Around Active Galactic Nuclei
}

\author{
Moshe Elitzur \\ University of Kentucky, moshe@pa.uky.edu \\ Gary J. Ferland \\ Ohio State University, gary@uky.edu
}

Follow this and additional works at: https://uknowledge.uky.edu/physastron_facpub

Part of the Astrophysics and Astronomy Commons, and the Physics Commons

Right click to open a feedback form in a new tab to let us know how this document benefits you.

\section{Repository Citation}

Elitzur, Moshe and Ferland, Gary J., "Radiation Pressure and Emission Clouds Around Active Galactic Nuclei" (1986). Physics and Astronomy Faculty Publications. 174.

https://uknowledge.uky.edu/physastron_facpub/174

This Article is brought to you for free and open access by the Physics and Astronomy at UKnowledge. It has been accepted for inclusion in Physics and Astronomy Faculty Publications by an authorized administrator of UKnowledge. For more information, please contact UKnowledge@lsv.uky.edu. 


\section{Radiation Pressure and Emission Clouds Around Active Galactic Nuclei \\ Digital Object Identifier (DOI) \\ http://dx.doi.org/10.1086/164226 \\ Notes/Citation Information \\ Published in The Astrophysical Journal, v. 305, no. 1, p. 35-44. \\ (c) 1986. The American Astronomical Society. All rights reserved. \\ The copyright holder has granted permission for posting the article here.}

This article is available at UKnowledge: https://uknowledge.uky.edu/physastron_facpub/174 
The ASTrophysiCAL Journal, 305:35-44, 1986 June 1

(1) 1986. The American Astronomical Society. All rights reserved. Printed in U.S.A.

\title{
RADIATION PRESSURE AND EMISSION CLOUDS AROUND ACTIVE GALACTIC NUCLEI
}

\author{
Moshe Elitzur \\ Department of Physics and Astronomy, University of Kentucky \\ AND \\ GARY J. FERLAND \\ Department of Astronomy, Ohio State University \\ Received 1985 July 24 ; accepted 1985 November 25
}

\begin{abstract}
Detailed calculations of the contribution of the trapped line photons to the overall pressure in line-emitting regions around active galactic nuclei are presented. They lead to the conclusion that radiation pressure is a significant factor in determining the stability of these clouds.

Subject heading: galaxies: nuclei
\end{abstract}

\section{INTRODUCTION}

The generally accepted picture for the broad-line emission regions in active galactic nuclei is that of clouds embedded in a hot, rarefied gas with the two phases coexisting in pressure equilibrium (e.g., Blumenthal and Mathews 1975; McCray 1979; Krolik, McKee, and Tarter 1981). The calculation of the pressure inside the line-emitting clouds is therefore an essential ingredient in determining their allowed, stable configurations. In addition to the thermal pressure, due to the kinetic motions of the gas particles, the line photons trapped inside the cloud also contribute to the overall pressure. The potential importance of this component was first pointed out in this context by Williams (1972). Subsequent studies of this effect were made by McKee and Tarter (1975), Mathews (1976), Weymann (1976), and Krolik (1979). In these investigations the column densities assumed were relatively small (less than a few $10^{21} \mathrm{~cm}^{-2}$ ), the clouds were fully ionized, and the line optical depths were mostly below $\sim 10^{5}$. However, the work of Kwan and Krolik (1981) and Weisheit, Shields, and Tarter (1981) showed that more realistic values for the columns of the emission regions are above $10^{22} \mathrm{~cm}^{-2}$, and an $I$ front therefore develops in the cloud. As a result of the neutral region that is formed, optical depths of allowed transitions can easily exceed $10^{6}$. Scattering in the line wings thus becomes important. Although this causes a fundamental difference in the calculation of radiation pressure, as we show below, this effect did not draw much attention in the more recent calculations of photoionization models.

Recently, however, we pointed out that radiation pressure may attain relatively large values in cloud interiors, and that it may be a significant factor in determining the stability of the emission regions (Ferland and Elitzur 1984; hereinafter paper I). The basic reason is that the total pressure at the edge of the cloud is simply gas pressure, while the radiation pressure deep inside the cloud is determined independently and can easily exceed the gas pressure. Thus, no stable solution with constant pressure is possible.

The aims of the present paper, a follow-up of Paper I, are twofold. The first one is to expand on the method and techniques used in calculating the radiation pressure inside the clouds. This is a somewhat delicate problem because of the complex nature of line radiative transfer at very large optical depths where scattering in the line wings is important. Section II of the paper is therefore devoted to a rather elaborate and extensive discussion of the formalism we use. Two different derivation methods for the final expression for the line radiation pressure at large optical depths are presented.

The other objective of the paper is to repeat the previous calculations incorporating the numerous developments that occurred since our first study. In addition to the constant, ongoing improvement in the atomic data base used in the model calculations, a number of other significant developments took place, in particular the two discussed by Wills, Netzer, and Wills (1985). The first of those is the realization that the continuous spectrum used in Paper I, following Kwan and Krolik, is somewhat harder than observations indicate. The second one is the improved treatment of the contribution of the Fe II ion to the cooling in neutral regions of the cloud. The net result of both of these effects is to lower the temperature of the neutral zone significantly; radiation pressure in this region thus becomes insignificant. However, the results for radiation pressure in the ionized zone are essentially unchanged from those of Paper I. We therefore corroborate our previous conclusion that radiation pressure may be a significant factor in determining what are the stable configurations of the emission-line clouds.

The model calculations are outlined in $\S$ III. The results are presented in $\S \mathrm{IV}$ and discussed in $\S \mathrm{V}$.

\section{RADIATION PRESSURE \\ a) Basic Considerations}

For radiation intensity $I_{v}$, the standard expression for the radiation pressure per unit frequency, $P_{v}$, is (e.g., Schwarzchild 1965)

$$
P_{v}=\frac{1}{c} \int I_{v} \mu^{2} d \Omega,
$$

where $\mu=\cos \theta$ and $\theta$ is the direction of propagation of the radiation. When the radiation field is isotropic, its pressure and energy density,

$$
U_{v}=\frac{1}{c} \int I_{v} d \Omega,
$$

are related through the familiar expression

$$
P_{v}=\frac{1}{3} U_{v} .
$$


This relation holds for a rather wide range of circumstances. If the angular distribution of $I_{v}$ is expanded in a power series in $\mu$, then only powers higher than the second will lead to violations of equation (3). However, the successive coefficients of this expansion are decreasing approximately like the optical depth (e.g., Schwarzchild 1965, p. 40), so deviations from equation (3) will only be proportional to $1 / \tau^{2}$. Hence, when the medium is optically thick at the frequency $v$ equation (3) is an excellent approximation for the radiation pressure.

The only radiative quantity we therefore need to know in order to calculate the radiation pressure is the angle-averaged flux, $J_{v}$, since

$$
U_{v}=\frac{1}{c} 4 \pi J_{v} .
$$

Combining equations (3) and (4) we find that $P(v)$, the overall contribution of the line to the radiation pressure integrated over frequency, is

$$
P(v)=\frac{4 \pi}{3 c} \int J_{v} d v .
$$

Let us now introduce the line width, $\Delta v$, through the definition

$$
\Delta v=\frac{1}{\bar{J}} \int J_{v} d v
$$

where

$$
\bar{J}=\int J_{v} \Phi(v) d v
$$

is the integrated mean intensity in the line and $\Phi(v)$ is the normalized line profile $\left[\int \Phi(v) d v=1\right]$. The quantity $\bar{J}$ is readily available in the escape probability approximation because it is related directly to the source function $S$ via

$$
\bar{J}=S\left(1-\beta_{e}\right),
$$

where $\beta_{e}$ is the photon escape probability (e.g., Castor 1970; see also Elitzur 1982; Rybicki and Hummer 1983; Rybicki 1984; Elitzur 1984). Equations (5)-(7) therefore enable us to calculate the contribution of any line to the pressure of the trapped radiation once the level populations are known, because the line source function is simply $B_{v}\left(T_{x}\right)$-the Planck function of the line excitation temperature (e.g., Mihalas 1978). Our final expression for $P(v)$ is therefore

$$
P(v)=\frac{4 \pi}{3 c} B_{v}\left(T_{x}\right) \Delta v\left(1-\beta_{e}\right) .
$$

Since the only lines that can make a significant contribution to the radiation pressure are the ones that are optically thick, the corresponding escape probabilities are much smaller than 1 (of order $1 / \tau$ ) and will be omitted from the expressions for the radiation pressure in the following discussion (but not from the actual calculations).

Equation (8) can be rearranged so that the radiation pressure is expressed as the thermal pressure of an equivalent gas of particles with kinetic temperature $T_{x}$ and density $n_{p}$, namely

$$
P(v)=n_{p} k T_{x} \text {. }
$$

The equivalent density, $n_{p}$, is given by

$$
n_{p}=n_{0} f(y),
$$

where

$$
n_{0}=\frac{8 \pi}{3 \lambda^{3}} \frac{\Delta v}{v}, \quad f(y)=\frac{y}{[\exp (y)-1]}, \quad y=\frac{h v}{k T_{x}},
$$

and $\lambda$ is the wavelength.

The expressions just derived show that when the temperature becomes large $\left(k T_{x}>h v ; y<1\right)$, the equivalent particle density $n_{p}$ approaches the limiting value $n_{0}$ and the pressure due to line radiation behaves just like ordinary particle pressure, increasing linearly with the excitation temperature. Notice the strong dependence of the equivalent particle density on the line wavelength, reflecting the larger photon phase space at higher frequencies (see, e.g., Elitzur 1982).

In contrast with the high-temperature limit, when the temperature is decreasing (so that $k T_{x}<h v ; y>1$ ) the equivalent particle density, and the radiation pressure, drops exponentially, reflecting the functional dependence of the Wein tail. Figure 1 plots the equivalent particle density $n_{p}$ as a function of the excitation temperature $T_{x}$ for various wavelengths in the optical and UV regimes and the same fractional line width of $\Delta v / v=10^{-4}$. The figure shows, for example, that a $3000 \AA$ optically thick line with an excitation temperature of $10,000 \mathrm{~K}$ and a fractional line width of $10^{-4}$ will contribute to the pressure as much as an additional gas component with a particle density of $10^{9} \mathrm{~cm}^{-3}$ and temperature $T_{x}$.

\section{b) The Line Width}

It is evident from equation (8) that the line width is a crucial parameter in the calculations since the line radiation pressure is directly proportional to it. For lines with a moderate optical depth $\tau$ (less than $\sim 10^{4}$ ) the damping wings are optically thin, and the line emission profile is essentially identical to its absorption profile (Thomas 1957; see also Mihalas 1978, p. 419). In this case $\Phi(v)$ is simply described by the Doppler profile $\pi^{1 / 2} \exp \left(-x^{2}\right)$, where $x=\left(v-v_{0}\right) / \Delta v_{\mathrm{D}}$ is the dimensionless frequency shift from line center and $\Delta v_{\mathrm{D}}=$ $(2 k T / m)^{1 / 2} v_{0} / c$ is the Doppler width. It is then easy to show that the line width is simply

$$
\Delta v=\Delta v_{\mathrm{D}} \times\left\{\begin{array}{ll}
(2 \pi)^{1 / 2} & \tau \lesssim 1 \\
2(\ln \tau)^{1 / 2} & \tau \gg 1
\end{array} .\right.
$$

The situation when the line optical depth exceeds $\sim 10^{4}$ is much more complicated because scattering in the damping wings becomes significant, and the frequency dependence of the emission profile is not known before the entire radiative transfer problem is solved. Fortunately, the problem of line radiative transfer at large optical depths has been treated in a number of independent studies and the solutions are available in the literature. These include works by Adams (1972), who solved the problem using the Feautrier method; by Harrington (1973), who solved the problem analytically in the large optical depth limit; and Monte Carlo calculations by Bonilha et al. (1979) and by Slater, Salpeter, and Wasserman (1982). All of these studies are in agreement with each other regarding the frequency distribution of the radiation field. They all provide expressions for various frequency half-widths, which in the limit of $a \tau \gg 1$ are given by (in dimensionless units)

$$
x=f(a \tau)^{1 / 3},
$$

where $a$ is the damping parameter $\left(4.7 \times 10^{-4}\right.$ for $\operatorname{Ly} \alpha$ at $10,000 \mathrm{~K}), \tau$ is the line center optical depth to the slab center 


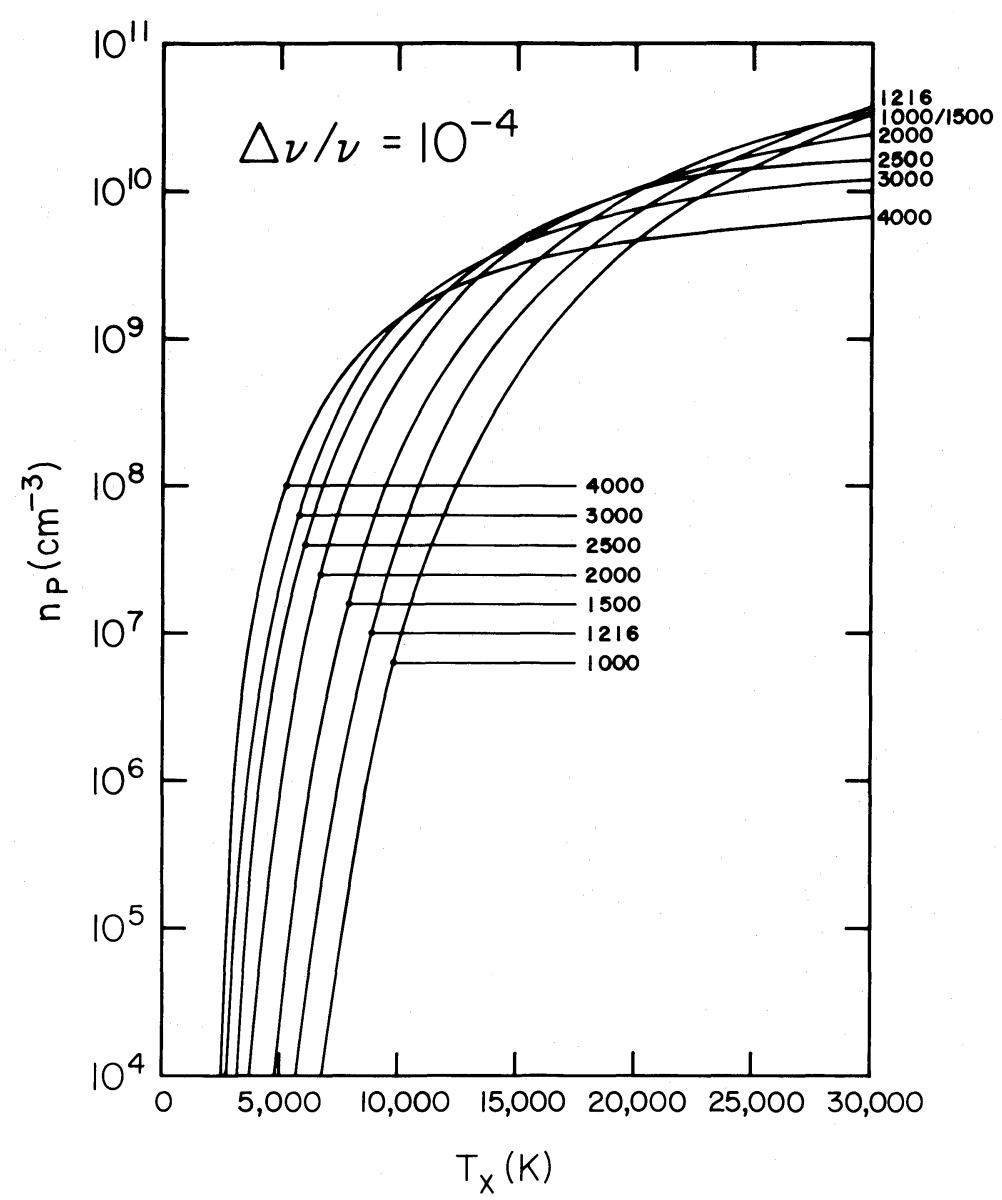

Fig. 1.-Equivalent particle density $n_{p}$ that provides kinetic pressure equal to radiation pressure, as a function of the excitation temperature for various wavelengths, as marked (in $\AA$ ). Fractional line width is $10^{-4}$ in all cases.

(the overall optical depth is $2 \tau$ ), and $f$ is a number of order unity.

Adams estimated the frequency where the average photon escapes and obtained $f=0.83$. Harrington obtained $f=1.07$ for the position of the maximum of the emergent flux (notice that Harrington reported his results using mean, rather than line center, optical depths). Using his figures, this appears to be very similar to the frequency width of the $J$ distribution at slab center. Bonilha et al. report a value of $f=1.24$ for the mean frequency shift of an escaping photon, essentially identical to what is obtained in the second Monte Carlo study of Slater et al. (notice that eq. [11] of Bonilha et al. contains a typographical error, corrected by Slater et al. following their eq. [23]).

The frequency width required here is the value that will provide a rectangular profile with the same area as the proper integral of the source function. An analysis of the profiles plotted in Figure 4 of Adams shows that an excellent approximation for this half-width is provided by equation (12) with $f=1.16$. We therefore adopt this value, and our expression for the full line width in the case of large optical depths $(a \tau \gg 1)$ is

$$
\Delta v=\Delta v_{\mathrm{D}} 2.3(a \tau)^{1 / 3} \text {. }
$$

An important point, evident from the plots provided by Adams for the source function as a function of frequency (his Fig. 3), is that the width of the frequency distribution varies very little with position in the slab. This is also evident from the mean intensity plots of Harrington, as mentioned above, and is a result of the strong coupling between distant regions caused by scattering in the line wings. We therefore use the expression provided in equation (13) for all locations in the slab, with $\tau$ being half the total slab thickness.

We note that in all of the studies mentioned above the line transfer problem was solved using photon injection in the central plane of a plane-parallel slab. However, Harrington presents also the solution for a uniform source distribution in the slab, and his results for both cases are essentially the same. Also, Bonilha et al. comment that the source can be either a point source or distributed over the midplane. It therefore appears safe to assume that the spatial distribution of the radiative source makes little difference to the resulting frequency distribution of the radiation field.

We note also that in spite of the large optical depths involved, the transfer problem was solved in these papers in the limit of slabs that are "effectively thin," namely-neglecting photon destruction and assuming that the only interaction of the radiation with matter is through photon scattering in the line. This assumption can potentially break down in the present situation because of two physical processes. The first of those is continuous absorption, since photons absorbed by an overlapping continuum leave the pool of line photons. However, the only continuous absorption that can interfere with line scattering in the objects of interest here is absorption by the Balmer continuum (we assume that there is no dust). But the optical depth at the Balmer edge is only $\sim 1$, many 
orders of magnitudes smaller than the large line optical depths where this discussion is relevant. Balmer continuum absorption will therefore compete with line scattering only at the extreme wings, at frequency shifts exceeding about $(a \tau)^{1 / 2}$, which are much larger than those contained in the range enclosed by expression (13). The effect of the overlapping continuum on the line width can therefore be neglected. However, it can be significant in determing the level populations, or, equivalently, the excitation temperatures because of the change in the number of scatterings before escape. This effect is properly taken into account in our calculations, as described below.

Collisional de-excitation can also break the assumption of pure scattering because a photon will be lost to the thermal pool before the radiative process can take place. This will happen when the density is high enough that the probability for collisional de-excitation, $C$, exceeds the probability for radiative escape $\beta_{e} A$, where $A$ is the Einstein coefficient for the transition. Because at large optical depths $\beta_{e}$ is essentially equal to $1 / \tau$, the "effectively thin" assumption breaks down when

$$
\tau \sim A / C
$$

Another way to derive the same relation is to note that the ratio of probabilities per unit time for collision and radiative decay for each atom is $C / A$. A collision will therefore occur after $N$ scatterings if $N C / A \approx 1$, and because the mean number of scatterings that a photon actually undergoes in the slab is approximately $\tau$, equation (14) follows again.

We see that once the line optical depth exceeds $\sim A / C$, a "thermalization limit" is encountered, and the assumption of a purely scattering nebula does not apply anymore. Therefore, in evaluating the optical depth for the line width expression (eq. [13]) we use the minimum of the actual optical depth of the line and the one prescribed by equation (14). We feel that this prescription provides a reasonable and conservative estimate for the effect of collisions on photon scattering. Admittedly, though, this is probably the most poorly understood part of the calculation.

The transfer calculations upon which we rely apply only to the case of resonance scattering because they use Hummer's (1962) type II redistribution function. The redistribution function appropriate for subordinate lines, Hummer's case IV, is considerably more complex, and we are not aware of any detailed calculations that have utilized it. One expects, however, that the subordinate line widths will be at least as large as in the case of resonance scattering because both the upper and lower levels are broadened by radiation damping. At any rate, this is a minor point because the only subordinate lines that are included in our calculations are the hydrogen Balmer lines, and they never provide the major contribution to the overall radiation pressure.

\section{c) An Alternative Derivation}

Because of the central role that the expression for the radiation pressure plays in our calculations, we provide now an alternative derivation for it. The approach utilized for this derivation is similar to the one used by Mathews (1976).

The line contribution to the radiation pressure can be written as

$$
P(v)=\frac{1}{3} N(v) h v,
$$

where $N(v)$ is the number density of line photons inside the cloud. The number of photons escaping the source per unit volume and unit time, $N_{\text {esc }}$, can be obtained from $N(v)$ by dividing it by the mean time that a photon spends in the source before escaping. If $L$ is the mean total path length traveled before escape, then this time scale is simply $L / c$, so $N_{\text {esc }}=$ $N(v) c / L$. But $N_{\text {esc }}$ is also equal to $\beta_{e} N_{2} A$, where $N_{2}$ is the population of the upper level. Equating the two expressions we find

$$
N(v)=\frac{g_{2} A n_{2} L}{c N_{\mathrm{sc}}},
$$

where we replaced $N_{2}$ with the population per sublevel, $n_{2}$, times $g_{2}$, the level statistical weight. We have also replaced the escape probability, $\beta_{e}$, with the inverse of $N_{\mathrm{sc}}$, the mean number of scatterings that a photon undergoes in the source before escape. The reason for doing this is that Bonilha et al. provide convenient analytic expressions for the quantities $L$ and $N_{\mathrm{sc}}$ (their $L_{0}$ and $N_{0}$ ). In the limit of large optical depths $(a \tau \gg 1)$ these become

$$
L_{0}=\tau(6.5 a \tau)^{1 / 3}, \quad N_{0}=1.6 \tau,
$$

where $L_{0}$ is in units of line center mean free path. Therefore, in order to convert $L_{0}$ to $L$, which is the actual length, it has to be divided by the absorption coefficient at line center

$$
\kappa_{0}=\sqrt{\pi} \frac{h v}{4 \pi \Delta v_{\mathrm{D}}} \frac{c^{2}}{2 h v^{3}} g_{2} A\left(n_{1}-n_{2}\right) .
$$

Combining equations (16)-(18) leads to

$$
N(v)=\frac{1}{h v} \frac{4 \pi}{c} B_{v}\left(T_{x}\right) 2.1 \Delta v_{\mathrm{D}}(a \tau)^{1 / 3}
$$

When this expression for the photon density is inserted in equation (15) for the radiation pressure, the relations derived in the previous section are recovered.

This analysis shows that a consistent expression for the line width is

$$
\Delta v=\Delta v_{\mathrm{D}} \sqrt{\pi} L_{0} / N_{0}
$$

where $L_{0}$ and $N_{0}$ are the expressions listed by Bonilha et al. For optical depths larger than five we use their equations (6) and (7), and for the smaller values we use their equations (8) and (9).

Notice that $E(v)$, the line emissivity per unit volume, is simply $N_{\text {esc }} h v$. The relation between line radiation pressure and line emissivity is therefore that of stored photons, $N(v)$, to escaping photons, $N_{\text {esc }}$, and

$$
P(v)=\frac{l}{3 c} E(v) \frac{L_{0}}{\tau}
$$

where $l$ is the thickness of the source.

\section{IONIZATION CALCULATIONS}

The radiation pressure can be calculated easily using the formalism described above, once the level populations and optical depths are known. The calculation of these quantities, assuming steady-state photoionization equilibrium, is outlined in this section. The ionization and thermal structures are calculated along traditional lines (see Williams 1967; Davidson 1972; MacAlpine 1972; Davidson and Netzer 1979; Kwan and Krolik 1981). Radiation impinges upon one side of a plane-parallel slab of gas, held at constant pressure (gas + radiation) by a postulated intercloud medium (see 
McCray 1979; Krolik, McKee, and Tarter 1981; Shull et al. 1985). The slab is divided into a set of thin zones, and the equations of statistical and thermal equilibrium are solved for each zone, given the optical depth-modified ionizing continuum and the constraint that the total gas and radiation pressure be kept constant. Line and continuum emission from each zone are calculated and added to the net spectrum.

The ionizing continuum we use is normalized to reproduce the typical observed X-ray to ultraviolet slope of $\alpha_{\mathrm{ox}} \approx 1.2$, as defined and measured by Zamorani et al. (1981). As such, this continuum is significantly softer than that used in our first paper (that work employed the continuum used by Kwan and Krolik 1981, which Wills, Netzer, and Wills 1985 note has roughly a factor of 6 too strong an X-ray component). The continuum used here extends to $100 \mathrm{keV}$, where only electron scattering is important and the Klein-Nishina cross section has started to roll over.

The present calculation includes all the physical processes coupling radiation and matter now known to be important (see Kallman and McCray 1982; Halpern and Grindlay 1980). Some details are discussed in the recent note by Netzer and Ferland (1984). A recent improvement (besides the constant advances in the atomic data base) is the use of the secondary ionization rates for suprathermal electrons calculated by Shull and van Sternberg (1985). Another improvement is our use of the simplified model of the Fe II ion suggested by Wills, Netzer, and Wills (1985). As noted by them, this simple three-level ion provides much more cooling than was obtained by ions which neglected line overlap. The net effect of this treatment of the Fe II ion, and the softer X-ray continuum mentioned above, is to produce neutral regions that are considerably cooler (by $\sim 10^{3}$ K) than we previously found.

Line transfer is treated in the escape probability formalism (see Netzer 1976; Davidson and Netzer 1979; Kwan and Krolik 1981). As suggested by Hubbard and Puetter (1985), we use escape probabilities with different functional forms for resonance versus subordinate lines. For resonance lines, such as Ly $\alpha$ or $C_{\text {IV }} \lambda 1549$, we use the incomplete redistribution escape probability chosen to reproduce the detailed transfer calculations (see $\S$ II)

$$
\beta(\tau)=\frac{1}{1+1.6 \tau} .
$$

For subordinate lines, and Lyman lines higher than Ly $\alpha$ (i.e., $\operatorname{Ly} \beta$ ) we use an escape probability given by the above for optical depths where only the core is active $\left(\tau \lesssim 10^{4}\right)$, and the following complete redistribution escape probability for larger optical depths (see Avery and House 1968; Hubbard and Puetter 1985)

$$
\beta(\tau)=\frac{1}{1+160 \tau^{1 / 2}}
$$

Note that in both these expressions $\tau$ is the line center optical depth to the slab center, for a total slab thickness of $2 \tau$.

All of the calculations presented here are the result of at least two iterations, a necessity if diffuse fields and line transfer are to be treated properly (see Osterbrock 1974). On the second (and higher) iteration we use an escape probability given by the average between the probability of escape to the two faces of the cloud. That is, if the total optical depth in a line through the entire cloud is $T$, and we are considering a point at a depth $t$ from the inner face, the escape probability used will be $\beta=[\beta(t)+\beta(T-t)] / 2$. On the second and latter iterations the diffuse fields of hydrogen and helium are treated by $\Lambda$ iteration rather than by modified on-the-spot methods. The processes of line fluorescence and continuum overlap are treated as in Elitzur and Netzer (1985) and Netzer, Elitzur, and Ferland (1985). These include the effects of He I and He II Ly $\alpha$ on the ionization rates, and the destruction of $\mathrm{Mg}$ II $\lambda 2798$ and $\mathrm{Fe}$ II photons by the Balmer continuum. In a separate study (Netzer, Elitzur, and Ferland 1985), these techniques were utilized to calculate the Bowen mechanism. The results produced were in excellent agreement with the previous calculations of Weymann and Williams (1969) and Kallman and McCray (1980).

Most atoms and ions are treated as two-level systems (i.e., a ground and ionized state), and most lines are treated in a twolevel approximation. Important exceptions are hydrogen (treated as a seven-level atom, plus continuum, with $2 s$ and $2 p$ considered independently) and $\mathrm{He}$ ( five triplet levels and continuum, basically as in Feldman and McAlpine 1978).

The solar composition used here is that of Lambert (1978) and Lambert and Luck (1978), with additions from references cited in Pagel and Edmunds (1981). The actual mixture, by number, is $\mathrm{He}: \mathrm{C}: \mathrm{N}: \mathrm{O}: \mathrm{Ne}: \mathrm{Mg}: \mathrm{Si}: \mathrm{S}: \mathrm{Ar}: \mathrm{Fe}: \mathrm{H}=1000: 4.7$ : $0.98: 8.3: 1.0: 0.42: 0.43: 0.17: 0.037: 0.33: 10^{4}$. The ionizing continuum used here, which we label the "standard continuum," is a two-component continuum similar to those used by Mushotzky and Ferland (1984) and Wills, Netzer, and Wills (1985):

$$
\frac{d N}{d E}=a E^{-\alpha 1} \exp \left(\frac{-E}{E_{0}}\right)+b E^{-\alpha 2} \text { photons } \mathrm{cm}^{-2} \mathrm{~s}^{-1} \mathrm{eV}^{-1}
$$

where we have chosen $\alpha 1=1.5$ (Oke, Shields, and Korycansky 1984) and $\alpha 2=1.7$ (Mushotzky 1982). The ratio of the normalization constants $a$ and $b$ was chosen to produce $\alpha_{\mathrm{ox}} \approx 1.2$. The cutoff value of $E_{0}$ was chosen to be 20 Ryd, so as not to exceed the observed soft X-ray flux. The motivation for this continuum is summarized by Wills, Netzer, and Wills (1985). The overall normalization is chosen to provide a prescribed ionization parameter $U$ which is the ratio of $\varphi / c$, where $\varphi\left(\mathrm{cm}^{-2} \mathrm{~s}^{-1}\right)$ is the surface density of ionizing photons, to the free electron density, all evaluated at the inner edge of the cloud.

The optical depth enters into the calculation of the radiation pressure in two different ways-both in the expression for the line width and in the factor $1-\beta_{e}$ which relates $\bar{J}$ and $S$ (eq. [7]). We have included a very large number of lines in the total radiation pressure. Many of these may not ever be optically thick, and all lines are optically thin near the edges of the cloud. Physically, the radiation pressure tends to zero near the edge of an ion's creation zone (where line photons are able to escape freely in one direction), but the two-sided escape probability tends to only half at the boundary (rather than one) if the medium is optically thick. This causes the radiation pressure to be overestimated. Accordingly, we choose the smaller of the inner and outer escape probabilities in the evaluation of $1-\beta_{e}$, as a conservative estimate of the total radiation pressure. As mentioned above, we use the smaller of the total optical depth or the thermalization length in the calculation of the line width.

$$
\text { IV. RESULTS }
$$

The model calculations described in the previous sections were performed for a wide range of parameters, similar to those 
believed typical of the conditions in the broad line emission clouds around active galactic nuclei.

A major difference from the results of Paper I is the lack of significant buildup of radiation pressure in the neutral zone. This is due to the lower temperature in the neutral region, a result of the somewhat softer continuous spectrum we use here as well as the enhanced $\mathrm{Fe}$ II cooling. On the other hand, the results for the radiation pressure in the ionized region are similar to those of Paper I.

An inspection of the detailed results shows that although the $C$ IV $\lambda 1549$ line makes a significant contribution to the radiation pressure, the main contribution comes from the trapped Ly $\alpha$ photons. This makes it easy to derive simply analytic expressions for the radiation pressure in the slab. Equation (8) can be written as

$$
P_{\mathrm{rad}}=\text { const } \frac{N_{2}}{N_{1}} \Delta v,
$$

where $N_{1}$ and $N_{2}$ are the populations of the first two hydrogen levels. Although there are quite a few physical processes affecting the populations of these levels, $N_{1}$ is determined in first order by the balance between photoionization and recombination, whereas the $n=2$ population is controlled mainly by balancing recombination against radiative decays to $n=1$. The result is that

$$
N_{2} / N_{1} \approx \text { const } N_{\mathrm{H}} U \tau_{\alpha},
$$

where $\tau_{\alpha}$ is the Ly $\alpha$ optical depth (the inverse of $\beta_{e}$ ) and $N_{\mathrm{H}}$ is the total hydrogen density, essentially equal to the electron density in the ionized zone. The line width, as shown above, is

$$
\Delta v=2.3 \Delta v_{\mathrm{D}}\left(a \tau_{w}\right)^{1 / 3}
$$

where

$$
\tau_{w}=\min \left(\tau_{\text {tot }}, 5 \times 10^{16} / N_{\mathrm{H}}\right)
$$

and $\tau_{\text {tot }}$ is the Ly $\alpha$ optical depth of the entire slab. Because the damping parameter $a$ is inversely proportional to $\Delta v_{\mathrm{D}}$, which varies as $T^{1 / 2}$, we find that

$$
\Delta v \propto\left(T \tau_{w}\right)^{1 / 3} .
$$

The final expression for the radiation pressure is therefore

$$
P_{\text {rad }}=\text { const } N_{\mathrm{H}} U \tau_{\alpha}\left(T \tau_{w}\right)^{1 / 3}
$$

and the result for $\beta=P_{\mathrm{rad}} / P_{\mathrm{gas}}$ is

$$
\beta \propto U \tau_{\alpha} \tau_{w}^{1 / 3} T^{-2 / 3} .
$$

Let us now check the variation of $\beta$ with position in the cloud for a given model. Our models are specified by the values of $U$ and $N_{\mathrm{H}}$ at the illuminated face of the slab and by the overall column density. The value of $\tau_{w}$ is therefore fixed throughout the cloud, and $\beta$ varies inside the slab in direct proportion to $\tau_{a}$, which in itself is linearly proportional to the column density of the neutral material $N_{n} R(R$ is the distance into the slab from the illuminated face). The ionization balance equation shows that in the ionized zone

$$
N_{n} / N_{\mathrm{H}} \propto \alpha / U \text {, }
$$

where $\alpha$ is the effective recombination coefficient, varying with temperature approximately as $T^{-0.8}$. The final expression for $\beta$ in the ionized zone is therefore

$$
\beta \propto N_{\mathrm{H}} R T^{-1.4},
$$

and because the temperature is fairly constant in this region, the radiation pressure should increase linearly with $R$. This result was first derived by Mathews (1976).

Figure 2 displays the variation of $\beta$ with $R$ for a model with an ionization parameter $U=10^{-1.75}$ and two different densities, as marked on the curves. The linear dependence of $\beta$ on $N_{\mathrm{H}} R$ in the ionized zone is evident; a good approximation for these models is $\beta=3.8 \times 10^{-22} N_{\mathrm{H}} R$. Once the $I$ front is reached, all the ionizing photons are absorbed and the $n=2$ level population is sustained mostly by collisions from the ground state. The $N_{2} / N_{1}$ ratio therefore declines considerably, leading to a sharp drop in the radiation pressure. Unlike the

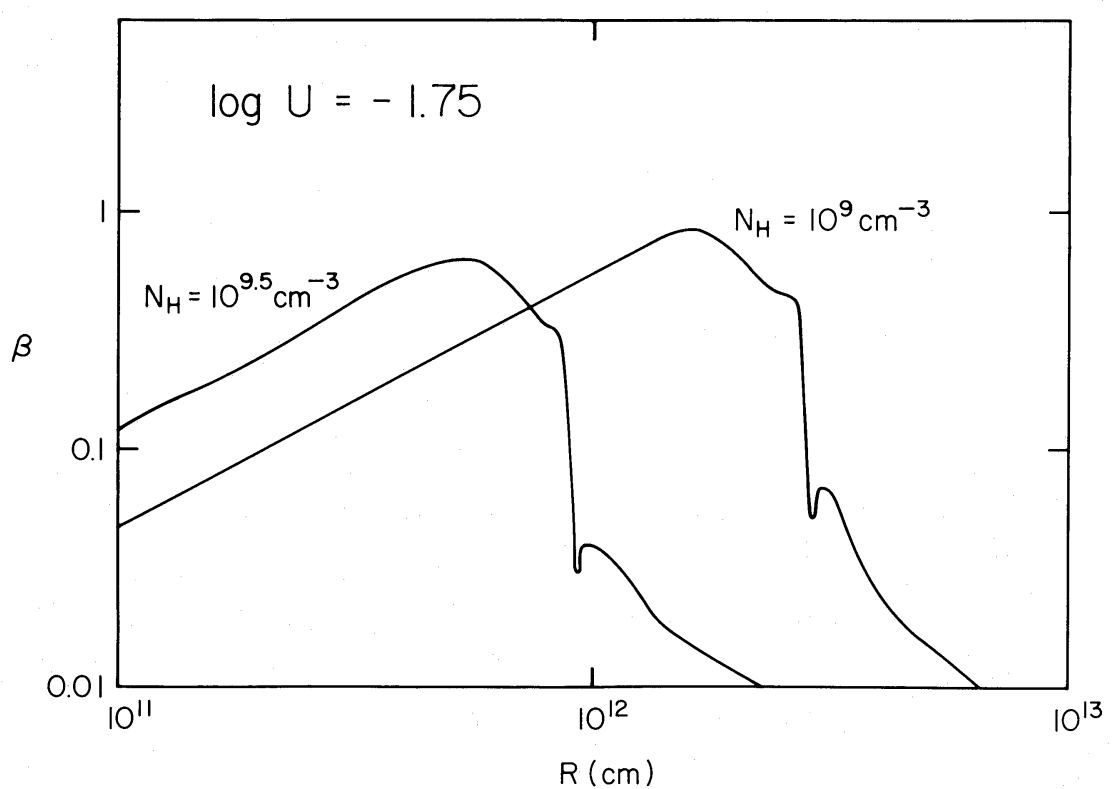

Fig. 2.-Ratio of radiation pressure to gas pressure as a function of depth into the cloud for an ionization parameter $10^{-1.75}$ and two particle densities, as marked. 
results of Paper I, no other ion makes a sizable contribution in the neutral zone, because of its lower temperature.

Let us now consider the maximum radiation pressure, $\beta_{\max }$, that can be obtained in a given cloud. Consider first the case that the overall column density is not too large, so an $I$ front does not develop and the material is fully ionized throughout the slab. The maximum radiation pressure will be obtained deep in the slab, so that in that location $\tau_{\alpha} \approx \tau_{w}=\tau_{\text {tot }}$. From equation (30) we therefore obtain

$$
\beta_{\max } \propto U \tau_{\text {tot }}^{4 / 3} T^{-2 / 3} .
$$

As before, $\tau_{\text {tot }}$ is proportional to the total neutral hydrogen column density, which in turn is proportional to $\alpha / U$ times the overall column density, $N_{\text {col }}$. This leads to the following final expression

$$
\beta_{\max } \propto N_{\text {col }}^{4 / 3} U^{-1 / 3} T^{-1.7},
$$

which holds as long as

$$
\tau_{\text {tot }}<5 \times 10^{16} \mathrm{~cm}^{-3} / N_{\mathrm{H}} \text {. }
$$

In Figure 3 we present the variation of $\beta_{\max }$ with the total column density $N_{\text {col }}$. Figure $3 a$ displays the results for a given ionization parameter and various particle densities. Because the temperature is a unique, slowly varying function of the ionization parameter, equation (34) predicts that $\beta_{\max }$ should be the same for these models, as long as equation (35) is obeyed. This is indeed the case. Figure $3 b$ displays the results for a set of models with the same particle density and different

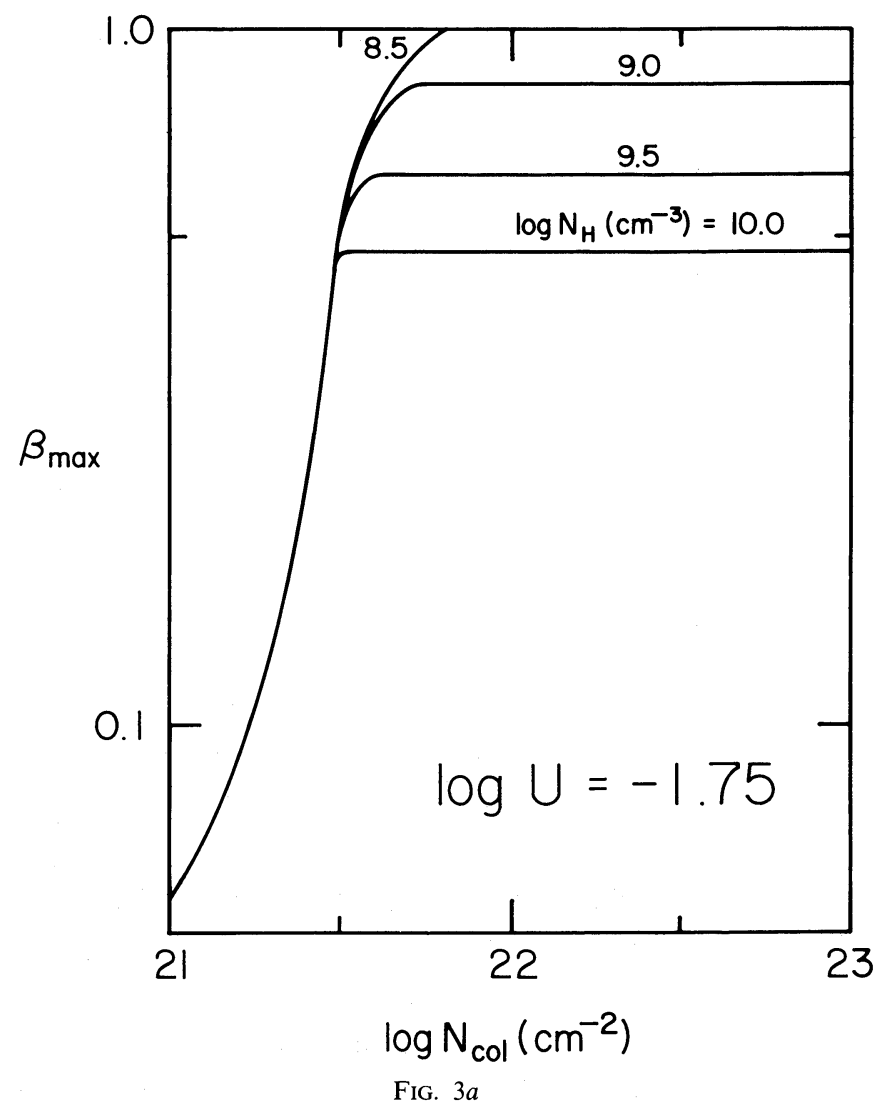

ionization parameters. For the same $N_{\text {col }}$, models with smaller $U$ have larger $\beta_{\max }$, in agreement with equation (34).

When the column density is further increased, all the ionizing photons are absorbed and an $I$ front develops. Increasing the column density further does not increase the maximum radiation pressure any more because, as shown above, the radiation pressure drops across the $I$ front. The maximum effective column, therefore, is that of the $I$ front, and it corresponds to the same optical depth $\left(\tau_{912} \approx 1\right.$ and $\tau_{\alpha} \approx$ few $\left.\times 10^{4}\right)$, irrespective of the values of $U$ and $N_{\mathrm{H}}$. Also, once a front is established, the total optical depth $\tau_{\text {tot }}$ immediately increases so that equation (35) does not hold anymore and $\tau_{w}$ is given by the thermalization limit $\left(\propto 1 / N_{\mathrm{H}}\right)$. From equation (30) we therefore obtain for this case

$$
\beta_{\max } \propto U N_{\mathrm{H}}^{-1 / 3} T^{-2 / 3} .
$$

This expression should apply to all slabs with total column density in excess of $\sim 10^{22} \mathrm{~cm}^{-2}$, which is the case for the broad line emitting clouds around active galactic nuclei.

Figure 4 displays the quantity $\beta_{\max }$ for models that developed an $I$ front. Figure $4 a$ presents the variation of $\beta_{\max }$ with $U$ for various fixed values of $N_{\mathrm{H}}$ that are marked on the curves. It is evident that the variation of $\beta_{\max }$ with $U$ is very nearly linear, as predicted by equation (36). Likewise, in Figure $4 b \beta_{\max }$ is plotted as a function of $N_{\mathrm{H}}$ for fixed $U$, and again, the agreement with the predicted $N_{\mathrm{H}}^{-1 / 3}$ behavior is reasonable. The slightly different power obtained can be attributed to the effect of collisions that modify the $N_{2} / N_{1}$ ratio.

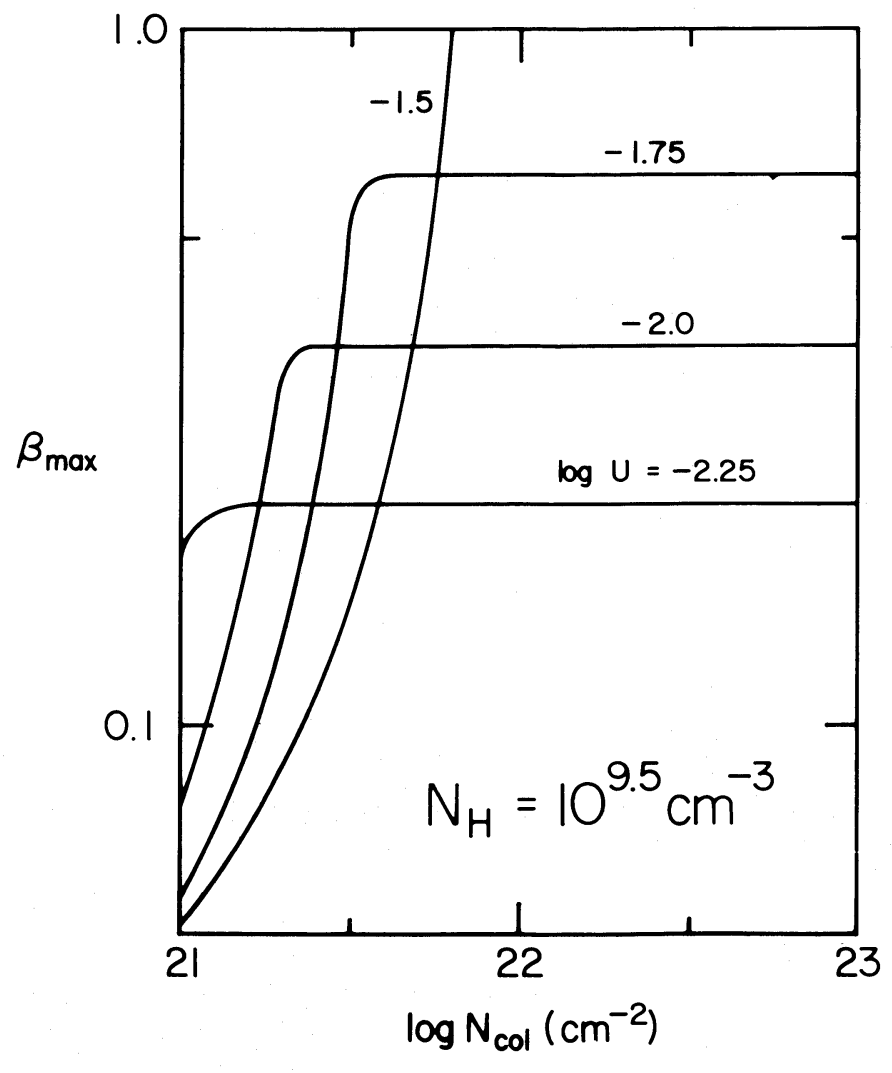

FIG. $3 b$

FIG. 3.-Maximum ratio of radiation pressure to gas pressure as a function of overall column density $(a)$ for fixed $U$ and various densities and $(b)$ for fixed $N_{\mathbf{H}}$ and various ionization parameters. 


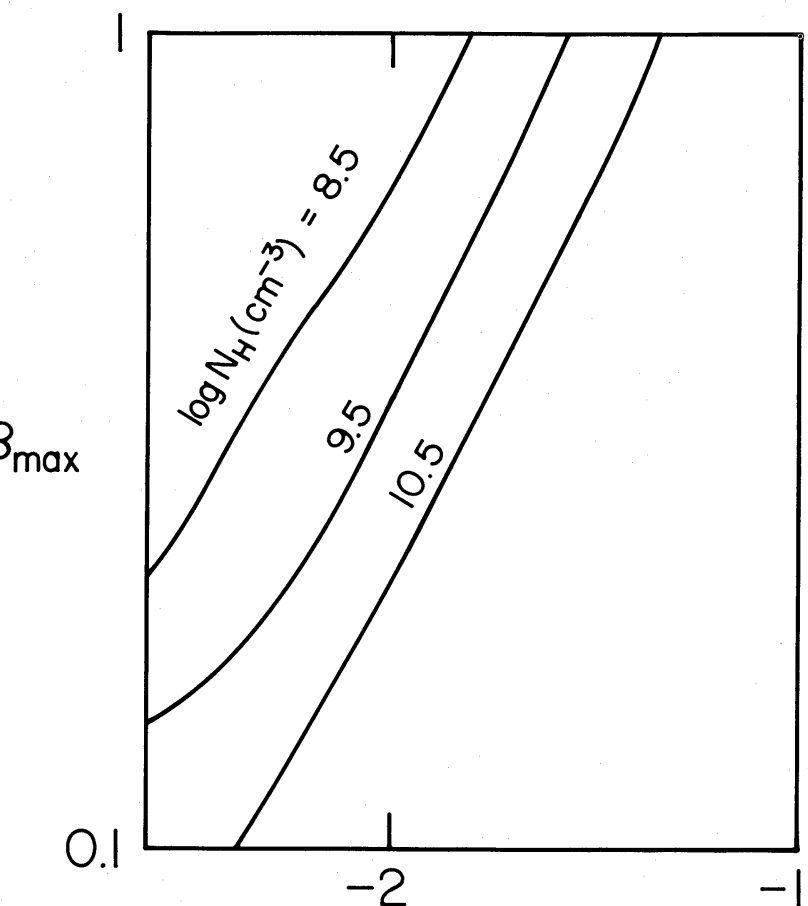

$\log U$

FIG. $4 a$

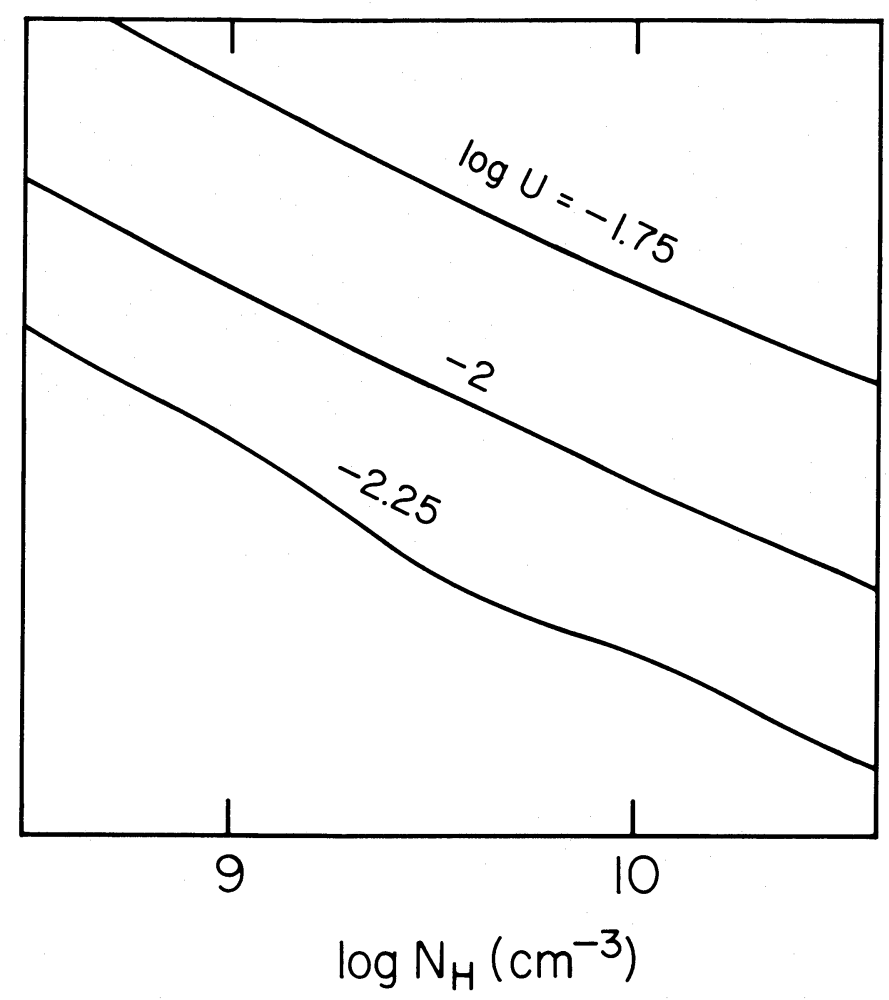

FIG. $4 b$

FIG. 4.- Maximum ratio of radiation pressure to gas pressure after the thermalization limit has been reached $(a)$ as a function of $U$ for fixed $N_{\mathrm{H}}$ and $(b)$ as a function of $N_{\mathrm{H}}$ for fixed $U$.

\section{DISCUSSION}

The results presented in the last section show that for certain combinations of the parameters, the radiation pressure in the cloud may exceed the gas pressure. Because the gas pressure is approximately constant throughout the ionized zone, and equal to the external, confining pressure, this means that a stable solution is not possible anymore because the pressure of the trapped line photons will disrupt the cloud. Notice that this is a direct consequence of the assumption of constant pressure

$$
P_{\mathrm{rad}}+P_{\mathrm{gas}}=\text { const }
$$

and it does not apply to other circumstances such as, for example, a stellar interior. In that case, the gradient of the total pressure is balanced by the gravitational force which is itself coupled to the overall structure of the object. In the present case, on the other hand, the gas pressure is uniquely determined by the conditions at the slab's boundary, where the pressure of the internally generated trapped radiation is negligible (since all the photons escape). However, in the inner zones the radiation pressure is determined by processes that are essentially independent of the requirements for hydrostatic equilibrium and may attain any value.

Combinations of $U$ and $N_{\mathrm{H}}$ that lead to $\beta_{\max }>1$ (approximately $U / N_{\mathrm{H}}^{1 / 3}>2.6 \times 10^{-5}$, where $N_{\mathrm{H}}$ is in $\mathrm{cm}^{-3}$ ) are therefore fobidden. In Figure 5 we plot the boundary of the allowed region of parameter space. Models that fall below the curve correspond to $P_{\text {rad }}>P_{\text {gas }}$ and are therefore unstable. We also present a boundary curve labeled " $\mathrm{KK}$ " obtained for the continuum used by Kwan and Krolik (1981). For the same combination of $U$ and $N_{H}$ the models with the standard continuum produce more radiation pressure than the KK contin-

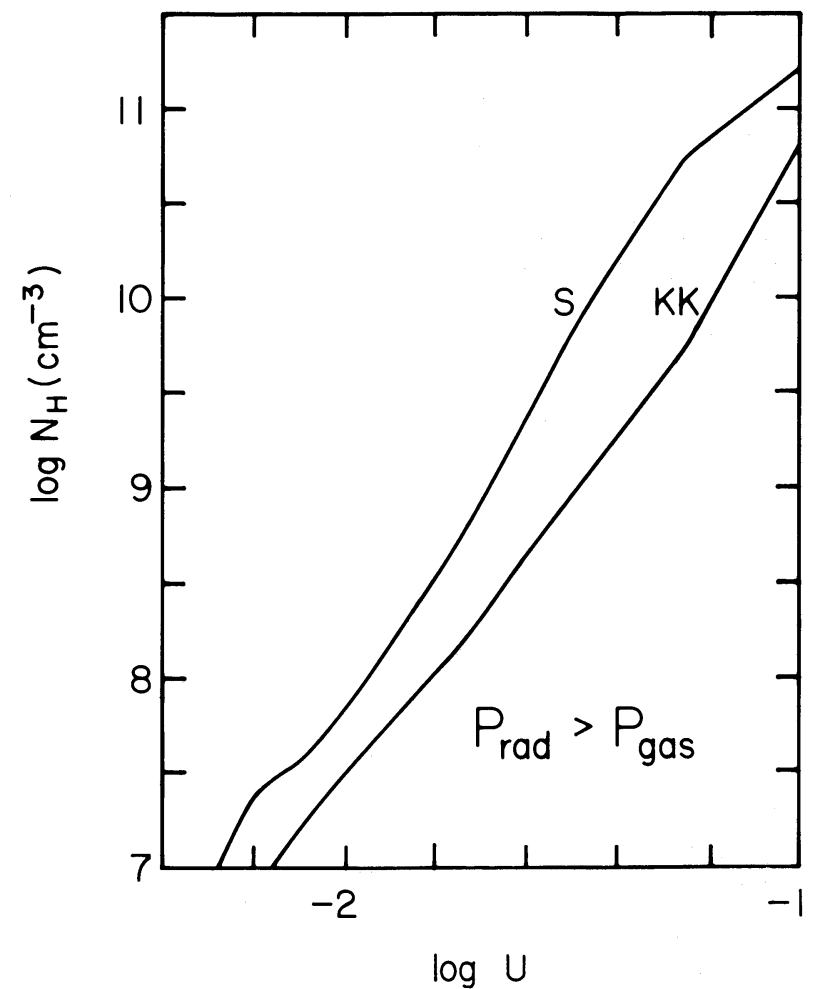

FIG. 5.-Density-ionization parameter diagram. Models in the lower right corner are unstable to disruption by internally generated radiation pressure. The curve labeled with " $S$ " corresponds to the boundary obtained with the standard continuum we use and the "KK" curve to the Kwan and Krolik (1981) continuum. 
uum. This is a consequence of the somewhat different ionization structure and temperature profile for the same model and the two continua. It is interesting to note that the Kwan and Krolik "standard" model $\left(U=10^{-1.5}, N_{\mathrm{H}}=10^{9.5}\right.$ $\mathrm{cm}^{-3}$ ) is allowed by the $\mathrm{KK}$ continuum, but forbidden by the standard continuum. We also note that other than a couple of Kinks, the KK boundary is rather similar to that of Paper I (Fig. 2) where the KK continuum was used.

The pressure of the trapped line photons therefore leads to a powerful constraint on the allowed stable configurations for the broad line emitting clouds around active galactic nuclei. Although the precise location of the boundary depends on somewhat uncertain details, such as the exact shape of the continuum spectrum, the fact that the curve passes near the values that are most commonly used for the emission regions strongly suggests that radiation pressure plays a significant role in determining the cloud parameters.

An additional constraint is provided by the requirement that the heating by a single photoionizing central source leads to hot and cold gas phases coexisting in pressure equilibrium (e.g., Krolik, McKee, and Tarter 1981; Guilbert, Fabian, and McCray 1983). This restricts the allowed range for the ionization parameter to a finite region. For example-for the KK continuum this approximate range is $10>U>10^{-1.3}$ (e.g., Paper I). Therefore, the intriguing possibility exists that steady state stability arguments alone may provide an explanation for the similarity in the values of $U$ and $N_{\mathrm{H}}$ among various sources. This, of course, has long been a puzzle in understanding the line-emitting regions around active galactic nuclei (e.g., Davidson and Netzer 1979).

As was mentioned above, the most uncertain part of our calculation is the effect of collisions on the line width. We have made the restrictive assumption that once collisions compete with scattering, the lines do not broaden anymore. This leads to a conservative, minimal estimate for the radiation pressure, and we feel that our results present a lower limit estimate for the significance of radiation pressure. However, it can be argued that collisions may actually broaden the line further. The possible consequences of larger line widths are discussed in the Appendix.

Our final comments are with regard to the possible role of the pressure of trapped radiation in other environments. Equation (10) shows that line radiation pressure varies with wavelength as $\lambda^{-3}$. Optical and UV lines are therefore more likely to play a significant dynamic role in various astronomical sources. One obvious environment is early-type stars. Indeed, calculations show that radiation pressure provides an adequate explanation for the winds in these stars (see, e.g., Abbott 1982, where an exhaustive list of references of earlier works can be found). However, the wind is driven in this case by the force of the direct stellar radiation rather than the pressure of the trapped photons. As shown by Castor (1974), the ratio of the former to latter is of order of the ratio of expansion and thermal velocities, which in early-type stars is $\sim 10^{3}$. We have performed numerical calculations and reached similar conclusions. Note, however, that in the late-type stars the initial expansion velocity, before dust grains form, is of order of the thermal velocity, so the pressure of the trapped line photons may be of importance there.

We have also checked the effect of radiation pressure in "typical" static models of an $\mathrm{H}$ II region and a planetary nebula. In both cases the radiation pressure becomes a significant fraction ( $\gtrsim 10 \%)$ of the gas pressure. Although the expansion of such regions will tend to decrease the radiation pressure, it should be noticed that this may be an important force in these sources.

We would like to thank R. C. Puetter for his perceptive comments on the manuscript. This work was supported in part by NSF grants AST-8305094 and AST-8304895.

\section{APPENDIX}

\section{THE COMPLETE REDISTRIBUTION LIMIT}

The referee has pointed out that the line width may be actually larger than the value we used. To understand this possible further broadening, notice that in the calculations that provided the justification for the $(a \tau)^{1 / 3}$ behavior of the width, the photons migrated to the line wings only through scattering, which was coherent in the atom frame at those frequencies. However, in the presence of some other processes that can introduce photons incoherently at the wings, it is possible that the lines will be broadened by additional amounts.

An example of such a process is line interlocking by interactions of atoms in the $n=2$ state with the H $\alpha$ radiation field, thereby reprocessing the Ly $\alpha$ photons through the third energy level. Because of the inherent spread in energies of the $n=2$ and 3 levels, the photons that will find their way back to the Ly $\alpha$ pool will be injected incoherently over the full Lorentzian profile. It is easy to show that the rate for interaction with the $n=2-3$ radiation field is $\sim 10^{-4}$ of the spontaneous $n=2 \rightarrow 1$ decay rate. Since the number of scatterings that a Ly $\alpha$ photon undergoes while migrating from the line core to its wings is $\sim 10^{4}$, reprocessing through $\mathrm{H} \alpha$ is a competing process that offers an effective mechanism for incoherent broadening of the Ly $\alpha$ line wings. This effect has not been calculated quantitatively, to the best of our knowledge, but here we wish to show the consequences of this type of effect upon BLR clouds.

If the lines broaden to their full LTE limit, i.e., the frequency where the optical depth becomes unity, then the line width is given by

$$
\Delta v=\Delta v_{\mathrm{D}} 2 \pi^{-1 / 4}(a \tau)^{1 / 2} \text {. }
$$

The thermalization limit in this case, the equivalent of equation (35), is now given by

$$
\tau_{\text {tot }}<\left(3 \times 10^{14} \mathrm{~cm}^{-3} / N\right)^{2} \text {. }
$$

As long as the density is lower than $10^{12} \mathrm{~cm}^{-3}$, which is always the case here, this optical depth is larger than the value given by equation (35), and thermalization is not important under these circumstances.

\section{(C) American Astronomical Society - Provided by the NASA Astrophysics Data System}


The escape probability is also different. In the case of complete redistribution, the escape probability goes over to the square root limit when the optical depth exceeds $10^{4}$ (namely, one should use eq. [23] instead of eq. [22]). However, since the maximum radiation pressure occurs at an optical depth of this order, the results for the two cases do not differ by much on this account.

Of greater interest is the line width because it is now proportional to $(a \tau)^{1 / 2}$. This width is typically 10 times larger than before, resulting in a factor of 10 more radiation pressure. Under these circumstances almost all clouds with " near-standard" parameters become unstable to disruption by radiation pressure.

The real significance of this point is not that the line width could be actually larger. Rather, it is that the width would grow without any bound associated with the column density, since the thermalization limit is not encountered under these circumstances. Since the value of $\tau_{W}$ is not subject to the thermalization limit anymore, then after an $I$ front is established, the value of $\beta_{\text {max }}$ becomes proportional to $U N_{\mathrm{col}}^{1 / 2} T^{-3 / 4}$. Hence, any model will eventually disrupt if its column density is large enough.

The implications of this possible situation are far-reaching. If the thermalization limit is applicable, then the column density can be increased without affecting $\beta_{\max }$ beyond a certain point. However, if this constraint is removed, then the radiation pressure increases indefinitely with column density. This then leads to a limit on the possible column density for any given value of $U$. It is perhaps significant that the relevant column densities $\left(\sim 10^{22}-10^{23} \mathrm{~cm}^{-2}\right)$ are similar to those derived for the emitting clouds. One could then speculate that additional constraints on the possible size scale of the emission clouds could be provided by dynamical considerations related to the issue of cloud formation. Together with the possible limit on the column density, this could lead to rather tight restrictions on the allowed cloud parameters.

It is likely that the actual situation regarding the line width lies somewhere between the two limits that we considered (coherent scattering vs. the full LTE limit), but this point has not yet been studied quantitatively. We hope that its importance will stimulate further studies.

Abbott, D. C. 1982, Ap. J., 259, 282.

Adams, T. F. 1972, Ap. J., 174, 439.

Avery, L. W., and House, L. L. 1968, Ap. J., 152, 493.

Blumenthal, G. R., and Mathews, W. G. 1975, Ap.J., 198, 517.

Bonilha, J. R. M., Ferch, R., Salpeter, E. E., Slater, G., and Noerdlinger, P. D. 1979, Ap. J., 233, 649.

Castor, J. I. 1970, M.N.R.A.S., 149, 111

. 1974, M.N.R.A.S., 169, 279.

Davidson, K. 1972, Ap.J., 171, 213.

Davidson, K., and Netzer, H. 1979, Rev. Mod. Phys., 51, 715.

Elitzur, M. 1982, Rev. Mod. Phys., 54, 1125. 1984, Ap. J., 280, 653.

Elitzur, M., and Netzer, H. 1985, Ap. J., 291, 464.

Feldman, F. R., and MacAlpine, G. M. 1978, Ap. J., 221, 486.

Ferland, G. J., and Elitzur, M. 1984, Ap. J. (Letters), 285, L11 (Paper I).

Guilbert, P. W., Fabian, A. C., and McCray, R. 1983, Ap. J., 262, 564.

Halpern, J. P., and Grinlay, J. E. 1980, Ap. J., 242, 1041.

Harrington, P. J. 1973, M.N.R.A.S., 162, 43.

Hubbard, E. N., and Puetter, R. C. 1985, Ap. J., 290, 394.

Hummer, D. G. 1962, M.N.R.A.S., 125, 21

Kallman, T., and McCray, R. 1980, Ap.J., 242, 615.

. 1982, Ap. J. Suppl., 50, 263.

Krolik, J. H. 1979, Ap. J., 228, 13.

Krolik, J., McKee, C. M., and Tarter, C. B. 1981, Ap. J., 249, 422.

Kwan, J., and Krolik, J. 1981, Ap. J., 250, 478.

Lambert, D. L. 1978, M.N.R.A.S., 182, 249.

Lambert, D. L., and Luck, R. E. 1978, M.N.R.A.S., 183, 79.

Mathews, W. G. 1976, Ap. J., 207, 351 .

McCray, R. 1979, in Active Galactic Nuclei, ed. C. Hazard and S. Mitton

(Cambridge: Cambridge University Press), p. 227.

\section{REFERENCES}

McKee, C. F., and Tarter, C. B. 1975, Ap. J., 202, 306.

Mihalas, D. 1978, Stellar Atmospheres (San Francisco: Freeman).

Mushotzky, R. F. 1982, Ap. J., 256, 92.

Mushotzky, R., and Ferland, G. J. 1984, Ap. J., 278, 558.

MacAlpine, G. M. 1972, Ap. J., 175, 11.

Netzer, H. 1976, M.N.R.A.S., 177, 473.

Netzer, H., Elitzur, M., and Ferland, G. J. 1985, Ap. J., 299, 752.

Netzer, H., and Ferland, G. J. 1984, Pub. A.S.P., 96, 593.

Oke, J. B., Shields, G. A., and Korycansky, D. G. 1984, Ap. J., 277, 64.

Osterbrock, D. E. 1974, Astrophysics of Gaseous Nebulae (San Francisco: Freeman).

Pagel, B. E. J., and Edmunds, M. G. 1981, Ann. Rev. Astr. Ap., 19, 77.

Rybicki, G. B. 1984, in Methods in Radiative Transfer, ed. W. Kalkofen (Cambridge: Cambridge University Press), p. 1.

Rybicki, G. B., and Hummer, D. G. 1983, Ap. J., 274, 380.

Schwarzschild, M. 1965, Structure and Evolution of the Stars (New York: Dover).

Shull, J. M., and van Steenberg, M. E. 1985, preprint.

Shull, J. M., et al. 1985 , Ap. J., in press.

Slater, G., Salpeter, E. E., and Wasserman, I. 1982, Ap. J., 255, 293.

Thomas, R. 1957, Ap. J., 125, 260.

Weisheit, J., Shields, G., and Tarter, C. 1981, Ap. J., 245, 406.

Weymann, R. 1976, Ap. J., 208, 286.

Weymann, R., and Williams, R. 1969, Ap.J., 157, 1201.

Williams, R. E. $1967, A p . J ., 147,556$.

. 1972, Ap.J., 178, 105.

Wills, B. J., Netzer, H., and Wills, D. 1985, Ap. J., 288, 94.

Zamorani, G., et al. 1981, Ap. J., 245, 357.

Moshe Elitzur: Department of Physics and Astronomy, University of Kentucky, Lexington, KY 40506

GARY J. FERLAND: Department of Astronomy, Ohio State University, Columbus, OH 43210 